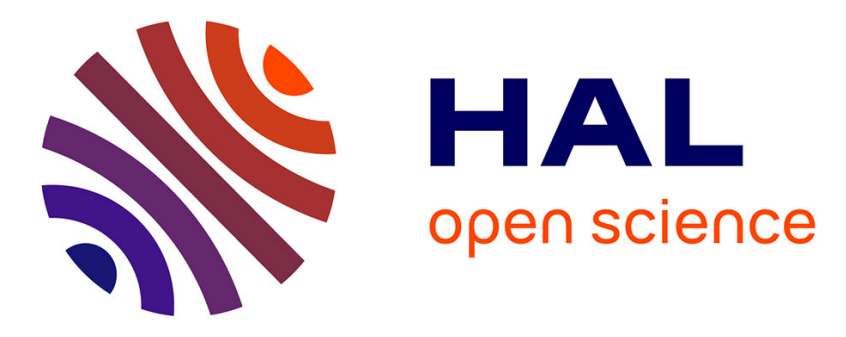

\title{
Design of Self-Supporting Surfaces with Isogeometric Analysis
}

Yang Xia, Angelos Mantzaflaris, Bert Jüttler, Hao Pan, Ping Hu, Wenping Wang

\section{- To cite this version:}

Yang Xia, Angelos Mantzaflaris, Bert Jüttler, Hao Pan, Ping Hu, et al.. Design of Self-Supporting Surfaces with Isogeometric Analysis. Computer Methods in Applied Mechanics and Engineering, 2019, 353, pp.328-347. 10.1016/j.cma.2019.05.030 . hal-02138360

\section{HAL Id: hal-02138360 https://hal.inria.fr/hal-02138360}

Submitted on 23 May 2019

HAL is a multi-disciplinary open access archive for the deposit and dissemination of scientific research documents, whether they are published or not. The documents may come from teaching and research institutions in France or abroad, or from public or private research centers.
L'archive ouverte pluridisciplinaire HAL, est destinée au dépôt et à la diffusion de documents scientifiques de niveau recherche, publiés ou non, émanant des établissements d'enseignement et de recherche français ou étrangers, des laboratoires publics ou privés. 


\title{
Design of Self-Supporting Surfaces with Isogeometric Analysis
}

\author{
Yang Xia ${ }^{\mathrm{a}, \mathrm{b}, *}$, Angelos Mantzaflaris ${ }^{\mathrm{c}, \mathrm{f}}$, Bert Jüttler ${ }^{\mathrm{d}, \mathrm{c}}$, Hao Pan ${ }^{\mathrm{e}}$, Ping $\mathrm{Hu}^{\mathrm{a}}$, Wenping Wang ${ }^{\mathrm{b}, *}$ \\ ${ }^{a}$ School of Automotive Engineering, State Key Laboratory of Structural Analysis for Industrial Equipment, Dalian \\ University of Technology, Dalian 116024, P.R. China \\ ${ }^{b}$ Department of Computer Science, The University of Hong Kong \\ ${ }^{c}$ Johann Radon Institute for Computational and Applied Mathematics (RICAM), Austrian Academy of Sciences \\ ${ }^{d}$ Institute of Applied Geometry, Johannes Kepler University, Linz, Austria \\ ${ }^{e}$ Microsoft Research Asia \\ ${ }^{f}$ Université Côte d'Azur, Inria Sophia Antipolis - Méditerranée, 2004 route des Lucioles, B.P. 93, 06902 Sophia \\ Antipolis, France
}

\begin{abstract}
Self-supporting surfaces are widely used in contemporary architecture, but their design remains a challenging problem. This paper aims to provide a heuristic strategy for the design of complex selfsupporting surfaces. In our method, non-uniform rational B-spline (NURBS) surfaces are used to describe the smooth geometry of the self-supporting surface. The equilibrium state of the surface is derived with membrane shell theory and Airy stresses within the surfaces are used as tunable variables for the proposed heuristic design strategy. The corresponding self-supporting shapes to the given stress states are calculated by the nonlinear isogeometric analysis (IGA) method. Our validation using analytic catenary surfaces shows that the proposed method finds the correct selfsupporting shape with a convergence rate one order higher than the degree of the applied NURBS basis function. Tests on boundary conditions show that the boundary's influence propagates along the main stress directions in the surface. Various self-supporting masonry structures, including models with complex topology, are constructed using the presented method. Compared with existing methods such as thrust network analysis and dynamic relaxation, the proposed method benefits from the advantages of NURBS-based IGA, featuring smooth geometric description, good adaption to complex shapes and increased efficiency of computation.
\end{abstract}

Keywords:

Masonry structure, Self-supporting, Isogeometric analysis, Equilibrium approach, Architectural geometry

\section{Introduction}

Masonry is a type of architecture built from bricks or stones that are laid in and bound together by mortar. It has been used for centuries and is regarded as one of the most important architectural

\footnotetext{
*Corresponding author

Email addresses: yangxia@dlut.edu.cn (Yang Xia), wenping@cs.hku.hk (Wenping Wang)
} 
forms. At present masonry is still widely used throughout the world, especially in low- and midrise buildings for its economical competitiveness and aesthetic value [1]. Masonry surfaces are a fascinating form of masonry that features a large structural span and various shapes, including arches, domes and vaults (see Fig. 1 for examples). The intrinsic characteristic of a masonry surface is that it is self-supporting, which means the surface bears the weight of the units, usually bricks or stones, and maintains the equilibrium state with minimum support only on the borders.

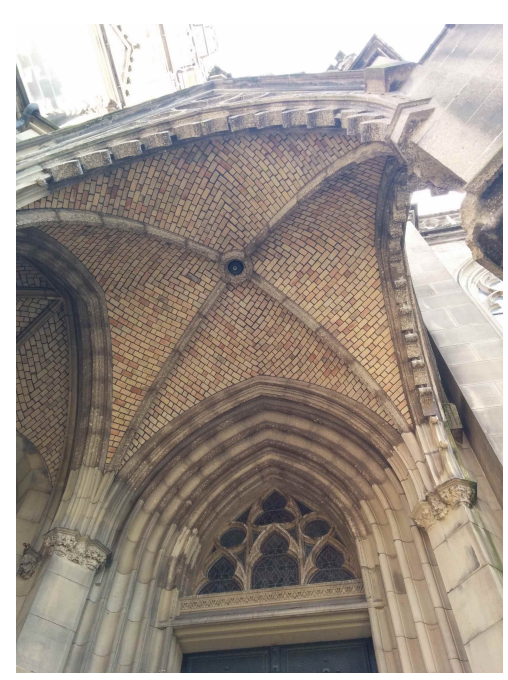

(a)

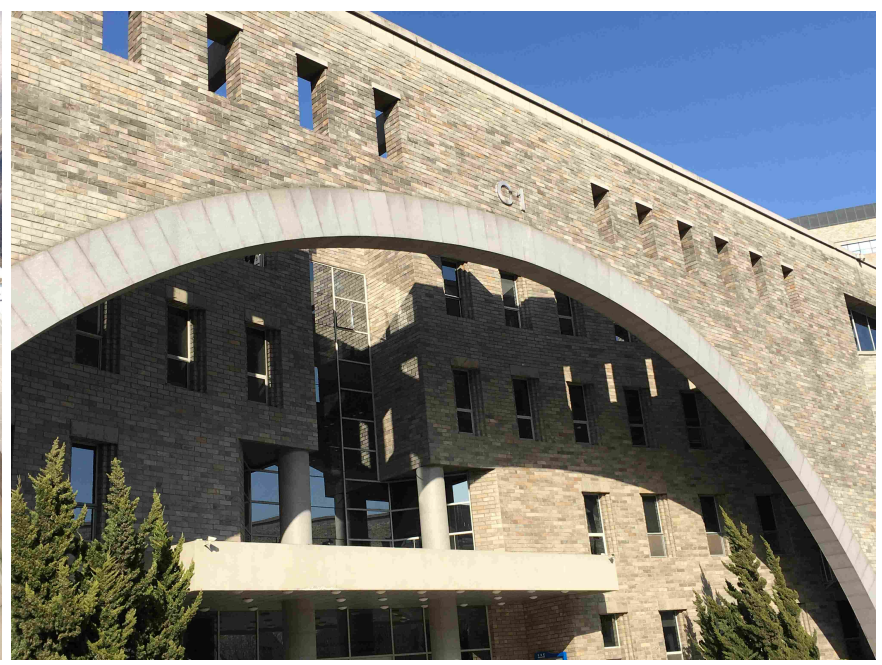

(b)

Figure 1: Masonry architectures with self-supporting surfaces. (a) A typical quadripartite vault at an entrance of Mariendom, Linz. (b) A modern building with a grand arch in Dalian.

Despite the long history and wide use of masonry structures, the design of self-supporting surfaces remains a challenge. The difficulty is rooted in the building material of masonry, which is composed of discrete units, such as stones or bricks. The units are held together almost all by themselves except for the constraint on the boundary to resist gravity, therefore the geometrical configuration needs to be carefully designed to keep the discrete units together and maintain the structure's shape. Robert Hook first discovered that the ideal shape of a masonry arch is analogous to an inverted catenary curve. This discovery has been applied to the design and assessment of masonry buildings, e.g., in Giovanni Poleni's study of St. Peter in the Vatican [2], and is still an influential guide to modern design [3]. Classical designs of self-supporting surfaces are based on empirical rules and various codes of practices, and the majority of contemporary designs are duplications of existing structures from history. However, contemporary architects have shown increasing interests in building new masonry structures with complex topology, which necessitates the development of new design methods.

We propose a heuristic strategy for the design of self-supporting masonry surfaces with complex geometry and topology. Specifically, we study the characteristic equilibrium state with the membrane shell theory. The Airy stress function is further used to transform the equilibrium equations into a single partial differential equation relating the Airy stress and the height function representing a self-supporting surface. Computationally, the nonlinear isogeometric analysis 
(IGA) method is applied for solving the equilibrium equation, in order to obtain the self-supporting surfaces. The IGA-based method produces an accurate solution with the convergence rate being one order higher than the degree of the NURBS basis used. To summarize, the contributions of this work are:

1. An equilibrium state modeled with IGA is proposed for self-supporting surfaces. Surfaces with complex topology are represented by multi-patch NURBS with high smoothness.

2. A heuristic strategy to design self-supporting surfaces is provided by tuning the Airy stress of the surfaces.

3. Newton's iteration is used to solve the nonlinear changes of gravity load caused by shape variation.

\subsection{Related work}

Equilibrium. The state of equilibrium is a fundamental consideration for designing selfsupporting structures. Influenced by the classic inverted catenary curve theory, numerical methods have been developed to describe the equilibrium state of self-supporting shapes. The thrust lines method (TLM) is proposed to consider the thrust lines as the centers of thrust forces. To maintain structural equilibrium, thrust lines must be contained within the masonry structure. Thrust line analysis is used to assess various masonry buildings [4] and constitutes the traditional equilibrium approach to calculate masonry structures [5]. Thrust network analysis (TNA), proposed by Block et al. $[6,7]$, assumes that a network of forces exists in the masonry and the structure is stable once the forces maintain equilibrium. The TNA method discretizes the surface into a network and models the equilibrium of the surface as the balance of forces on the network nodes. TNA enables the modeling of complex masonry structures [8], based on which form-finding and design algorithms have been proposed [9]. Liu et al. [10] define a new parameterization for the space of self-supporting triangular meshes, based on which the force distributions can be optimized. An optimal assembling process of building blocks is proposed by Deuss et. al. [11] to guide the physical construction of self-supporting structures. Bletzinger et al. studied the simulation of stress states of masonry structures and the numerical methods for simulation are merged into the form-finding and structural optimization [12-14].

Geometric representation. Triangular or polygon meshes are used in TLM and TNA methods, which have considerable discretization errors in shape representation. Miki et al. [15] introduced NURBS for the geometric representation of masonry structures. The spline-based geometric description represents an advantage over the mesh-based models used in TNA-related methods because they have better smoothness and more regular geometry variation.

Material. A masonry structure is composed completely of bricks or stone blocks held together by external forces, typically gravity. It can resist large compressive stress caused by its own gravity and outer loads but has almost no resistance to tension force [16]. Performing an accurate analysis on this type of bimodulus material is still a challenging problem in mechanics $[17,18]$. In classical assumptions on the behavior of masonry structures, the complex material property of masonry is simplified and is considered as the ideal tension-free elastic material that does not fail under pressure $[19,20]$. 
Isogeometric analysis. Isogeometric analysis (IGA) was first proposed by Hughes et al. [21] as a numerical simulation method to link the computer-aided design and simulation. In the present work, IGA is used for simulating self-supporting shapes for two benefits. First, in IGA the smooth geometry defined by NURBS describes the masonry surface more accurately. Second, IGA offers a rigorous framework for simulating the mechanics of masonry and the equilibrium state of the surface, which is a distinct advantage over the network-based TNA methods. Simulation of shell structures with IGA has been widely investigated to calculate the inner stress state of shells under outer loads [22-24]. Cazzani et al. applied a NURBS based isogeometric beam model to simulate the historical masonry arches [25]. Bletzinger et al. pioneered the research on designing selfsupporting surfaces for architecture design using isogeometric analysis. They applied the isogeometric B-Rep to structural analysis and form-finding of structural membranes, and proposed the updated reference strategy for form-finding with isogeometric membrane and beam elements [2629]. The design of architectural membranes with isogeometric elements is proposed in [26] for the first time, proposing the updated reference strategy for the form-finding of membranes. In [28] this method is expanded to form-finding of complex models described by untrimmed and trimmed multi-patch geometries, as summarized in [27]. A special isogeometric Bernoulli beam element embedded into a membrane structure was proposed in [29] for analyzing curves in the structure. The dynamic relaxation method is applied for masonry design [30]. In these works the isogeometric concept is used along with the form-finding algorithm to design self-supporting surfaces. The form-finding is the inverse problem of classical structural analysis, and the main challenge in form-finding is the handling of a singular stiffness matrix. In the present paper we also apply the isogeometric analysis for structure simulation by proposing a direct design strategy to compute the stresses in the masonry structure. Our method is effective in obtaining self-supporting shapes compared with the form-finding strategy.

\subsection{Outline}

This paper is organized as follows. In Section 2, we introduce the theory of self-supporting structures. In Section 3, we first present the heuristic design method using admissible Airy stress states, and then construct the computational model based on IGA. In Sections 4 and 5, results and discussions are given. Finally Section 6 concludes the paper.

\section{Self-supporting membrane shell theory}

\subsection{Geometry}

Three-dimensional shell structures are generally represented by their middle surfaces [31]. Based on Monge's description (Figure 2), a surface

$$
S=\{(x, y, h(x, y):(x, y) \in U)\}
$$

can be represented as a height function $h(x, y)$ over a two-dimensional (2D) domain $U$ with Cartesian coordinates $x$ and $y$ :

$$
\mathbb{R} \supset \begin{array}{ccc}
U & \rightarrow & \mathbb{R} \\
(x, y) & \rightarrow & h(x, y)
\end{array}
$$




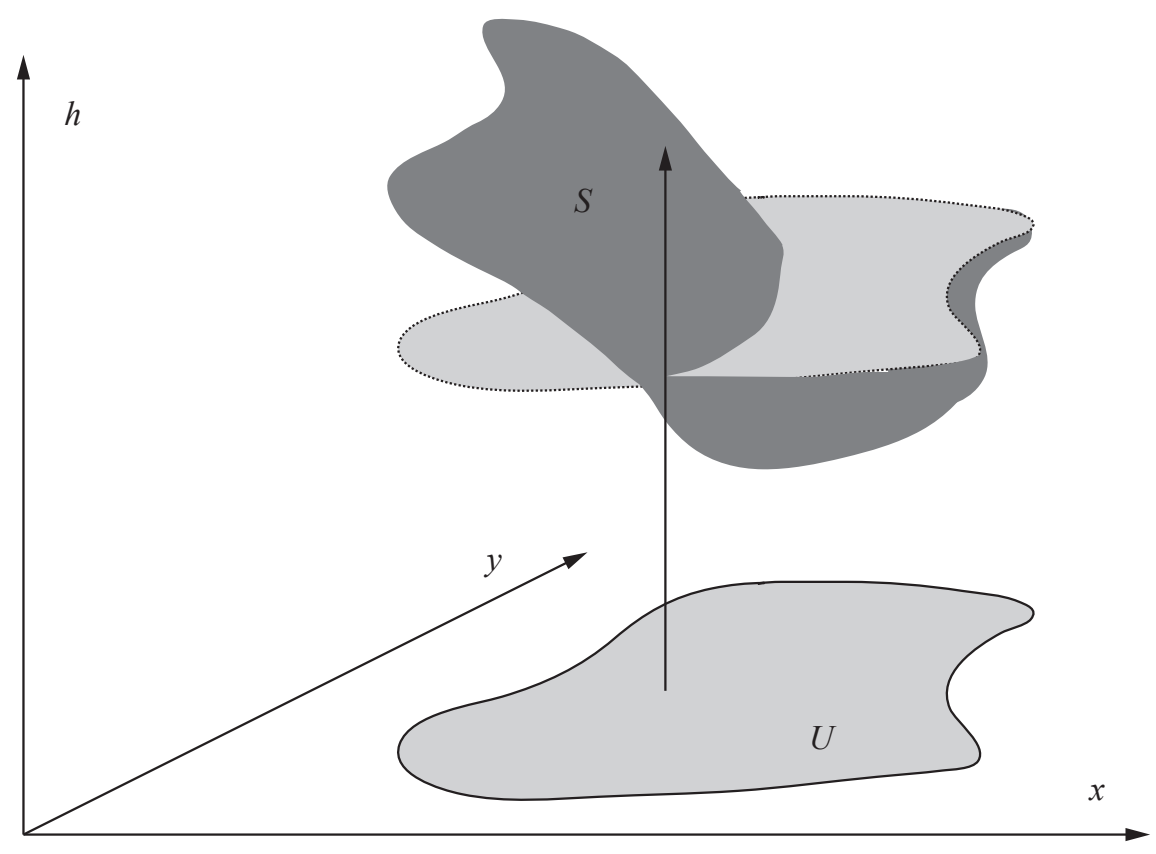

Figure 2: Monge's description of a masonry shell surface.

With this formulation, it is assumed that the masonry structure under consideration does not have any overhangs.

With IGA the 2D physical geometric domain $U$ and the height function $h(x, y)$ are both described by the same set of NURBS basis functions. In particular, a tensor product parameter domain $\Omega$ is set for each NURBS patch by the knot vectors. Denote the knot intervals in each of the parametric dimensions as $\left(\xi_{1}, \xi_{2}\right)$ and $\left(\eta_{1}, \eta_{2}\right)$, and we have $\Omega$ as $\left(\xi_{1}, \xi_{2}\right) \times\left(\eta_{1}, \eta_{2}\right)$. The geometry mapping using NURBS links the physical and parameter domains:

$$
x=\sum_{i=1}^{n} \sum_{j=1}^{m} \mathrm{R}_{i, j}(\xi, \eta) x_{i, j} \quad y=\sum_{i=1}^{n} \sum_{j=1}^{m} \mathrm{R}_{i, j}(\xi, \eta) y_{i, j}
$$

where $(x, y) \in U,(\xi, \eta) \in \Omega, R_{i, j}$ represents the 2-D NURBS bases and $x_{i, j}, y_{i, j}$ denotes the coordinates of control points, and $n$ and $m$ are the number of control points in the two directions. The geometry mapping in Eq. 2 defines a parameterization of the physical domain $U$ over the parameter domain $\Omega$.

Hence, the height function $h$ over the physical domain $U$ in Eq. 1 is also parameterized by $(\xi, \eta) \in \Omega$. Here we assume that the geometric mapping between parameter space and physical space is invertible. Furthermore, we assume that the height function $h(x, y)$ is given in the following NURBS form over the parameter domain.

$$
h=\sum_{i=1}^{n} \sum_{j=1}^{m} \mathrm{R}_{i, j}(\xi, \eta) h_{i, j}
$$

where $R_{i, j}$ represents the 2-D NURBS bases and $h_{i, j}$ denotes the height of the control points. $n$ and $m$ are the number of control points in the two directions. To calculate the derivatives of height to $x$ 
and $y$, the geometry mapping in Eq.2 is used. Conceptually, the height function can be expressed as

$$
h=\sum_{i=1}^{n} \sum_{j=1}^{m} \mathrm{R}_{i, j}(\xi(x, y), \eta(x, y)) h_{i, j}
$$

\subsection{Equilibrium analysis with membrane shell theory}

Membrane theory is used to describe the balance conditions relating the surface geometry and the stress field on the surface which resists external loads $[19,32]$. The stress per surface point when projected onto the horizontal plane is encoded by the symmetric $2 \times 2$ matrix

$$
\sigma=\left[\begin{array}{ll}
\sigma_{11} & \sigma_{12} \\
\sigma_{12} & \sigma_{22}
\end{array}\right]=\left[\begin{array}{cc}
\bar{N}_{x} & \bar{N}_{x y} \\
\bar{N}_{x y} & \bar{N}_{y}
\end{array}\right]
$$

where $\bar{N}_{x}, \bar{N}_{y}$ and $\bar{N}_{x y}$ are the projected stress forces in the normal and shear directions respectively. The shell membrane equilibrium can be expressed by the following second-order differential equations [33]:

$$
\begin{gathered}
\frac{\partial \sigma_{11}}{\partial x}+\frac{\partial \sigma_{12}}{\partial y}=0 \\
\frac{\partial \sigma_{12}}{\partial x}+\frac{\partial \sigma_{22}}{\partial y}=0 \\
\sigma_{11} \frac{\partial^{2} h}{\partial x^{2}}+2 \sigma_{12} \frac{\partial^{2} h}{\partial x \partial y}+\sigma_{22} \frac{\partial^{2} h}{\partial y^{2}}=-\bar{P}_{z}
\end{gathered}
$$

where $\bar{P}_{z}$ is the gravitational load on the surface

$$
\bar{P}_{z}=P_{z} \sqrt{1+\left(\frac{\partial h}{\partial x}\right)^{2}+\left(\frac{\partial h}{\partial y}\right)^{2}}
$$

$P_{z}=\rho g t$ is the unit area load, where $\rho$ is the density of the material, $g$ is the gravitational acceleration, and $t$ is the thickness of the surface. Assuming a uniform material density, without loss of generality, we use a constant $P_{z}=1$ for subsequent discussions. Eq. 6 represents the horizontal equilibrium, which is equivalent to the stress tensor $\sigma$ being divergence free. Eq. 7 represents the vertical equilibrium under external load.

For a simply connected domain, if there exists a function $\Phi(x, y)$ such that

$$
\sigma_{11}=\frac{\partial^{2} \Phi}{\partial y^{2}}, \quad \sigma_{22}=\frac{\partial^{2} \Phi}{\partial x^{2}}, \quad \sigma_{12}=-\frac{\partial^{2} \Phi}{\partial x \partial y},
$$

the divergence free property of $\sigma$ (Eqs. 6) is satisfied. The function $\Phi(x, y)$ is called the Airy stress function, as discussed in Ref. [34] (page 26, Chapter 2). Substituting Eq. 9 into Eq. 7, we obtain the following equilibrium equation in the vertical direction:

$$
\frac{\partial^{2} h}{\partial y^{2}} \frac{\partial^{2} \Phi}{\partial x^{2}}-2 \frac{\partial^{2} h}{\partial x \partial y} \frac{\partial^{2} \Phi}{\partial x \partial y}+\frac{\partial^{2} h}{\partial x^{2}} \frac{\partial^{2} \Phi}{\partial y^{2}}=-\bar{P}_{z}
$$


The equilibrium equation can be transformed into a dimensionless form. Let $L_{0}$ be the reference length. The coordinates $x$ and $y$ and the thickness of surface $t$ can be expressed by their dimensionless forms $x^{*}, y^{*}$ and $t^{*}$.

$$
x=x^{*} L_{0}, \quad y=y^{*} L_{0}, \quad h=h^{*} L_{0}, \quad t=t^{*} L_{0}
$$

The resultant stresses can be divided by $L_{0} P_{z}$ to give the dimensionless forms,

$$
\sigma_{22}^{*}=\sigma_{22} /\left(L_{0} P_{z}\right), \quad \sigma_{12}^{*}=\sigma_{12} /\left(L_{0} P_{z}\right), \quad \sigma_{11}^{*}=\sigma_{11} /\left(L_{0} P_{z}\right)
$$

The dimensionless form of equilibrium equation is

$$
\sigma_{22}^{*} \frac{\partial^{2} h^{*}}{\partial y^{* 2}}+2 \sigma_{12}^{*} \frac{\partial^{2} h^{*}}{\partial x^{*} \partial y^{*}}+\sigma_{11}^{*} \frac{\partial^{2} h^{*}}{\partial x^{* 2}}=-\sqrt{1+\left(\frac{\partial h^{*}}{\partial x^{*}}\right)^{2}+\left(\frac{\partial h^{*}}{\partial y^{*}}\right)^{2}}
$$

With the dimensionless form, the influence of stress states on the corresponding shapes can be clearly demonstrated. Therefore in the following solution with IGA, $L_{0}$ and $P_{z}$ are set as unit values and the generality of the resulting shapes is not affected. This is useful in choosing the parameters of Airy stress functions for the design of masonry surfaces.

An issue related to Eq. 9 is that, Eq. 6 is more general than Eq. 9, because divergence free stress tensors exist that are not second-order differentials of a function. Indeed, by the Hodge decomposition theorem [35], a divergence free vector field may contain an additional component of a harmonic vector field which may not be integrable on a domain with high genus. Nevertheless, the present approach focuses on designing the Airy stress function which is able to describe a wide range of stress states in masonry structures.

\subsection{Self-supporting condition of masonry structures}

In this work, we assume that the material of masonry reacts elastically to arbitrary compression but cannot bear the slightest traction. Therefore, the elasticity theory of masonry can be characterized by the requirement that, in addition to the above equilibrium equations, the resultant stress tensor $\sigma$ be negative semi-definite to ensure compression only:

$$
\begin{aligned}
\frac{\partial^{2} \Phi}{\partial x^{2}}+\frac{\partial^{2} \Phi}{\partial y^{2}} & \leq 0 \\
\frac{\partial^{2} \Phi}{\partial x^{2}} \frac{\partial^{2} \Phi}{\partial y^{2}}-\left(\frac{\partial^{2} \Phi}{\partial x \partial y}\right)^{2} & \geq 0
\end{aligned}
$$

In other words, the self-supporting condition implies that $\Phi$ is concave [32].

\section{Designing and computation self-supporting surfaces}

\subsection{Designing self-supporting surfaces by tuning Airy stress}

The Airy stress and height functions are the two unknowns to be solved in the design of selfsupporting surfaces. With the prescribed Airy stress, equilibrium in the horizontal directions is 
fulfilled automatically. The unknown left is the height function of the masonry structure, which should meet the equilibrium condition in the vertical direction. Designing various self-supporting shapes can thus be achieved by adjusting the Airy stresses. The problem to be solved is formulated as follows: given the planar boundary curves of a shape, find a smooth surface with a specific inner stress state, such that the self-supporting equilibrium under the given loads and boundary conditions is fulfilled. The design strategy is illustrated in Fig. 3.

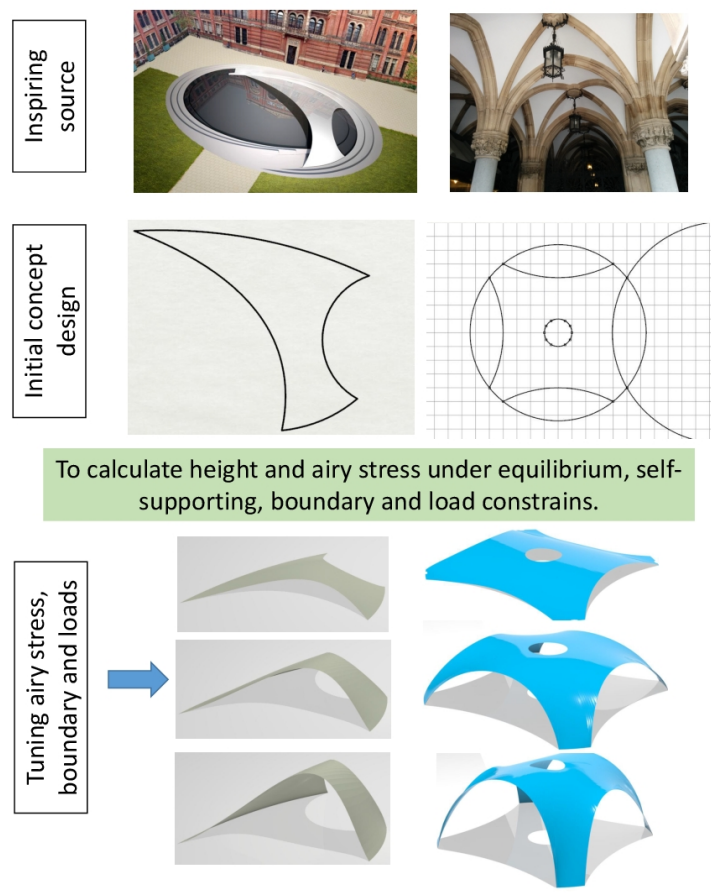

Figure 3: General problem description and design strategy. The sources of inspiration for the initial design shown in the picture include the sculpture "Crest" designed by Zaha Hadid Architects, and an indoor masonry vault roof. The picture of Crest is credited by the Zaha Hadid Architects.

Usually, the initial designs are simple curves that represent the boundaries of architectures and sculptures. With the proposed design strategy, the Airy stress function is prescribed, for which a particularly useful case is when the resultant stresses throughout the masonry shell are uniform [33], so that all points in the structure have the same inner stress and are equally strong for a single material. Such a situation represents an optimal state, as the structure can resist the largest total load without exceptional weak parts. The Airy stress function corresponding to the constant resultant stress state can be described as

$$
\Phi=\frac{1}{2} \sigma_{22}^{c} x^{2}+\frac{1}{2} \sigma_{11}^{c} y^{2}-\sigma_{12}^{c} x y
$$

where $\sigma_{11}^{c}, \sigma_{12}^{c}$ and $\sigma_{22}^{c}$ are constants. The corresponding stress tensor is

$$
\sigma=\left[\begin{array}{ll}
\sigma_{11}^{c} & \sigma_{12}^{c} \\
\sigma_{12}^{c} & \sigma_{22}^{c}
\end{array}\right]
$$


A linear polynomial $c_{1} x+c_{2} y+c_{3}$ can be added to the Airy stress function with arbitrary coefficients $c_{i}, i=1,2,3$. Because the linear term does not affect the obtained stress, it is omitted. The concave condition of the Airy stress function can be easily obtained by assigning values such that $\sigma_{11}^{c}+\sigma_{12}^{c} \leq 0$ and $\sigma_{11}^{c} \sigma_{12}^{c}-\sigma_{22}^{c}{ }^{2} \geq 0$ (Eq. 12). If $\sigma_{12}^{c}$ is set to zero, then the normal Airy stresses represent the major stress states, which can give meaningful directions for the design.

\subsection{Computational modeling of self-supporting surfaces with IGA}

Following the IGA framework, the non-uniform rational B-spline (NURBS) is used to describe the geometry of a self-supporting surface in the sense of Eq. 4. The shapes of masonry structures, such as domes and vaults, are generally composed of smooth surfaces, so can be accurately described by NURBS.

The designed planar footprint of the target self-supporting surface is given as input; During design computation, the footprint remains fixed and only the B-spline coefficients are varied (Eq. 3). Depending on its complexity, the footprint domain is described by single or multiple NURBS patches. For example, in the "Crest" model, the input geometry is described by a single NURBS patch (Fig. 4(a)). The "Tent" model consists of multiple patches (Fig. 4(b)), where neighboring patches share control points along their common boundaries, which makes the whole shape always continuous. Through this setting the continuity of the multi-patch surface is $C^{0}$, which suits for the design of masonry structures because kinks widely exist in the masonry buildings (see Fig. 1(a) for an example). To achieve higher order continuity, the algorithms in Refs. [36-38] can be used.

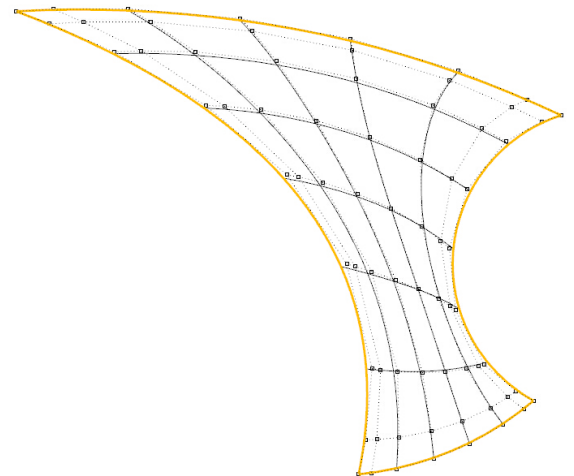

(a) Crest

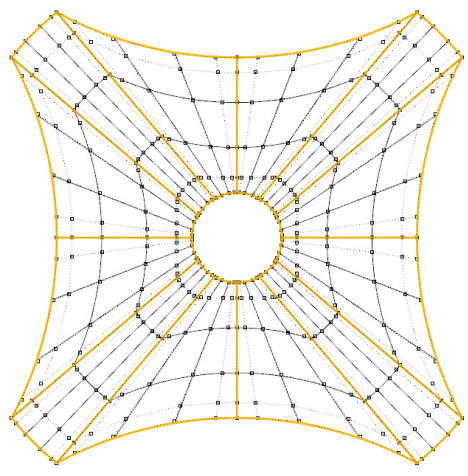

(b) Tent

Figure 4: The input NURBS surface for calculating two masonry structures. The crest model is described by a single patch and the tent model by multiple patches.

The equilibrium equation (Eq. 10) is a second-order partial differential equation. NURBS based IGA [21] is used to solve the equation and calculate the self-supporting surfaces. A selfsupporting surface generally has fixed or free boundary conditions, which can be easily implemented in the formulation of IGA by assigning Dirichlet boundary conditions. Permanent and static loads, especially the gravity, are deemed as Neumman boundary condition and discretized as the right-hand-side forces in IGA. The advantage of using IGA for computing masonry equilibrium is its high precision, for modeling various self-supporting shapes with different topological 
and geometric characteristics and boundary conditions. In particular, with IGA we construct the weak form of Eq. 10:

$$
\begin{aligned}
& {\left[\sigma_{11} \int_{\Omega_{e}} \frac{\partial \mathbf{R}}{\partial x} \frac{\partial \mathbf{R}}{\partial x} \mathrm{~d} \Omega+2 \sigma_{12} \int_{\Omega_{e}} \frac{\partial \mathbf{R}}{\partial y} \frac{\partial \mathbf{R}}{\partial x} \mathrm{~d} \Omega+\sigma_{22} \int_{\Omega_{e}} \frac{\partial \mathbf{R}}{\partial y} \frac{\partial \mathbf{R}}{\partial y} \mathrm{~d} \Omega\right] \cdot \mathbf{h}^{e}} \\
& =-P_{z} \int_{\Omega_{e}} \mathbf{R} \sqrt{1+\left[\left(\frac{\partial \mathbf{R}}{\partial x} \cdot \mathbf{h}^{\mathbf{e}}\right)^{2}+\left(\frac{\partial \mathbf{R}}{\partial y} \cdot \mathbf{h}^{\mathbf{e}}\right)^{2}\right]} \mathrm{d} \Omega
\end{aligned}
$$

Here, referring to Equations 3 and 4, we have

$$
\left[\begin{array}{l}
\frac{\partial \mathbf{R}}{\partial x} \\
\frac{\partial \mathbf{R}}{\partial y}
\end{array}\right]=\left[\begin{array}{ll}
\frac{\partial x}{\partial \xi} & \frac{\partial y}{\partial \xi} \\
\frac{\partial x}{\partial \eta} & \frac{\partial y}{\partial \eta}
\end{array}\right]^{-1}\left[\begin{array}{l}
\frac{\partial \mathbf{R}}{\partial \xi} \\
\frac{\partial \mathbf{R}}{\partial \eta}
\end{array}\right]
$$

Eq. 15 can be formulated into the following standard form:

$$
\mathbf{K} \cdot \mathbf{h}^{\mathbf{e}}=\mathbf{f}^{\mathbf{e}}
$$

where

$$
\begin{gathered}
\mathbf{K}=\sigma_{11} \int_{\Omega_{e}} \frac{\partial \mathbf{R}}{\partial x} \frac{\partial \mathbf{R}}{\partial x} \mathrm{~d} \Omega+2 \sigma_{12} \int_{\Omega_{e}} \frac{\partial \mathbf{R}}{\partial y} \frac{\partial \mathbf{R}}{\partial x} \mathrm{~d} \Omega+\sigma_{22} \int_{\Omega_{e}} \frac{\partial \mathbf{R}}{\partial y} \frac{\partial \mathbf{R}}{\partial y} \mathrm{~d} \Omega \\
\mathbf{f}^{\mathbf{e}}=-P_{z} \int_{\Omega_{e}} \mathbf{R} \sqrt{1+\left[\left(\frac{\partial \mathbf{R}}{\partial x} \cdot \mathbf{h}^{\mathbf{e}}\right)^{2}+\left(\frac{\partial \mathbf{R}}{\partial y} \cdot \mathbf{h}^{\mathbf{e}}\right)^{2}\right]} \mathrm{d} \Omega
\end{gathered}
$$

\section{pat}

\subsection{Iterative solution via Newton's method}

In Eq. 16, the force vector $\mathbf{f}^{\mathbf{e}}$ changes with the height vector $\mathbf{h}^{\mathbf{e}}$, which makes the equation nonlinear. We use Newton's method to solve the equation. According to Newton's method, the unbalanced force is defined as

$$
\mathbf{F}=\mathbf{K} \cdot \mathbf{h}^{\mathbf{e}}-\mathbf{f}^{\mathbf{e}}
$$

Then Eq. 16 is equivalent to $\mathbf{F}=\mathbf{0}$, which is solved through the iteration formulated as

$$
\left(\frac{\partial \mathbf{F}}{\partial \mathbf{h}^{\mathbf{e}}}\right)^{(\ell)} \cdot\left(\mathbf{h}^{\mathbf{e}^{(\ell+1)}}-\mathbf{h}^{\mathbf{e}(\ell)}\right)=-\mathbf{F}\left(\mathbf{h}^{(\ell)}\right)
$$

where $\ell$ is the iteration number, and ()$^{\ell}$ represents the value in the $\ell^{\text {th }}$ iteration. The derivative of $\mathbf{F}$ with respect to the height vector is

$$
\frac{\partial \mathbf{F}}{\partial \mathbf{h}^{\mathbf{e}}}=\mathbf{K}-P_{z} \int_{\Omega_{e}}\left(1+\left[\left(\frac{\partial \mathbf{R}}{\partial x} \cdot \mathbf{h}^{\mathbf{e}}\right)^{2}+\left(\frac{\partial \mathbf{R}}{\partial y} \cdot \mathbf{h}^{\mathbf{e}}\right)^{2}\right]\right)^{-\frac{1}{2}} \mathbf{R}\left(\left(\frac{\partial \mathbf{R}}{\partial x} \cdot \mathbf{h}^{\mathbf{e}}\right) \frac{\partial \mathbf{R}}{\partial x}+\left(\frac{\partial \mathbf{R}}{\partial y} \cdot \mathbf{h}^{\mathbf{e}}\right) \frac{\partial \mathbf{R}}{\partial y}\right) \mathrm{d} \Omega .
$$


The initial solution $\mathbf{h}^{(0)}$ is set as the planar footprint of the model. The stop condition is that the module of the unbalanced force $\mathbf{F}$ is smaller than a threshold value, which is generally set as $10^{-12}$ within the present paper.

In the formulation, the distributed body force (e.g. gravity) is already considered. Additional concentrated and distributed forces in the vertical direction can also be easily applied. Assume that a concentrated force $P$ is applied on the surface and is located to the point of patch $\Omega_{e}$ with parametric coordinates $\left(\xi_{p}, \eta_{p}\right)$. The force $P$ is then distributed to the control points of the patch, by adding $\int_{\Omega_{e}} R\left(\xi_{p}, \eta_{p}\right) P \mathrm{~d} \Omega$ to the right-hand-side of Eq. 16 .

\section{Results}

Without loss of generality, the footprints of the models are constrained in a simple square domain with the scale as $[-1,1] \times[-1,1]$. The loads are set as unit. Note that the dimensionless form of equilibrium function does not limit the generality of the present design algorithm.

\subsection{Validation with the analytic catenary surface}

A catenary is the curve that an idealized hanging chain assumes under its own weight if it is supported only at its ends. A catenary surface is the sweep surface of a catenary curve along a line perpendicular to the curve's plane. This surface is useful in the design of cathedrals and in Gothic arches used in Gothic architecture, to assure that no bending force is generated in the structure. Without loss of generality, for the catenary surface aligned with coordinate axes, the normal resultant stresses take uniform values and the shear resultant stresses vanish. Therefore, its self-supporting equilibrium equation is

$$
-\left(\frac{\partial^{2} h}{\partial x^{2}}+\frac{\partial^{2} h}{\partial y^{2}}\right)=\sqrt{1+h_{, x}^{2}+h_{, y}^{2}}
$$

The analytic solution to this equation is the catenary surface

$$
\bar{h}=-\cosh (x)+c
$$

where $c$ is an arbitrary constant. For example, setting $c=\cosh (2)$, we have

$$
\bar{h}=-\cosh (x)+\cosh (2)
$$

Fig. 5d illustrates the corresponding catenary surface. To validate our method, we compute the surface numerically using our proposed framework. The input coefficients are set according to Eq. 20 as $\sigma_{11}=-1, \sigma_{22}=-1$, and $\sigma_{12}=0$. The corresponding Airy function is $\Phi=-\frac{1}{2} x^{2}-\frac{1}{2} y^{2}$. The boundary curves of the catenary surface are set according to Eq. 22 and fixed as Dirichlet boundary conditions, as shown in Fig. 5a. The footprint on the horizontal plane forms a simple square over domain $(x, y), x=(-1,1), y=(-1,1)$, that is used as the initial solution for Newton's iteration (Sec. 3.3). Fig. 5c shows the shape obtained, which is almost the same as the analytic result as the ground truth. To measure the distance between the numerical result and the analytic 


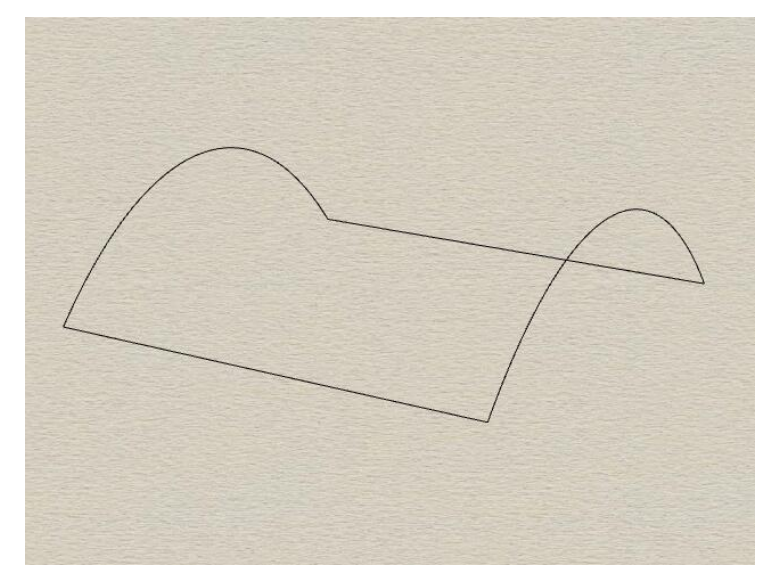

(a) Boundary curves

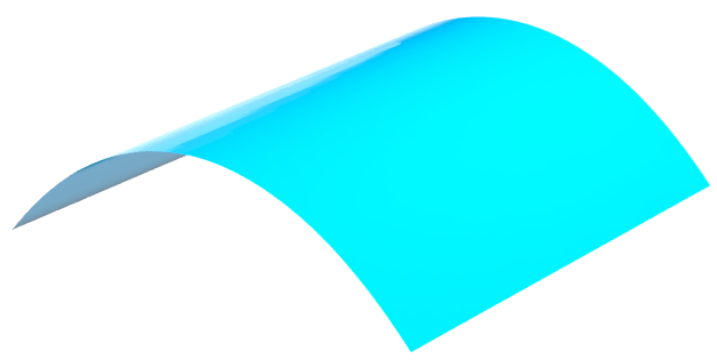

(c) Designed Result

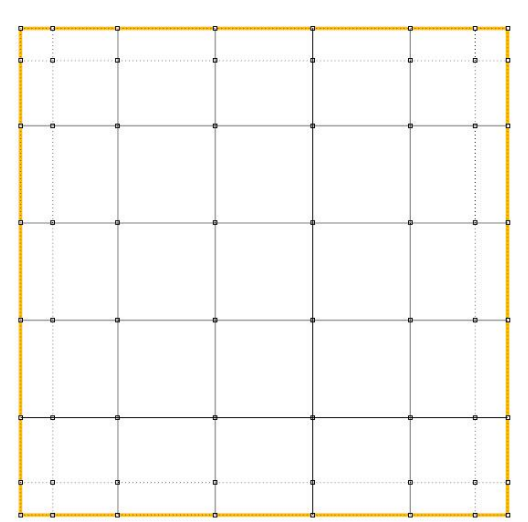

(b) Footprint domain

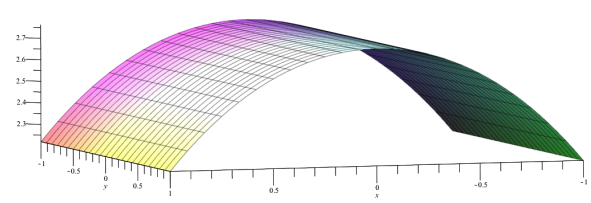

(d) Analytical solution

Figure 5: The validation of our solver for the catenary model. (a) The boundary curves are specified according to concept of catenary surface (b) The square NURBS patch of the footprint is used as the initial surface of the Newton iteration (c) The designed result (d) The analytical solution as ground truth for comparison.

surface, the $L_{2}$ error is computed:

$$
E_{L_{2}}=\sqrt{\int_{\Omega}(h-\bar{h})^{2} d \Omega}=\sqrt{\sum_{i=1}^{1000 \times 1000}\left(h_{i}-\bar{h}_{i}\right)^{2}}
$$

In the error computation, $1000 \times 1000$ evaluation points are evenly distributed over the horizontal footprint, $h_{i}$ and $\bar{h}_{i}$ are the calculated and the analytic heights respectively. We tested with increased polynomial degrees for NURBS ( $p$-refinement) and refined spline with more control points ( $h$-refinement), and show the errors in Table 1 . The convergence rate is further visualized in Fig. 6: for each model described by splines with degree $p$, the convergence rate of the $L_{2}$ error with respect to the step size (distance between two neighboring control points) is $p+1$. Our method can obtain a surface with $L_{2}$ error $9.48 \times 10^{-4}$ in $5.07 \times 10^{-3}$ seconds. 


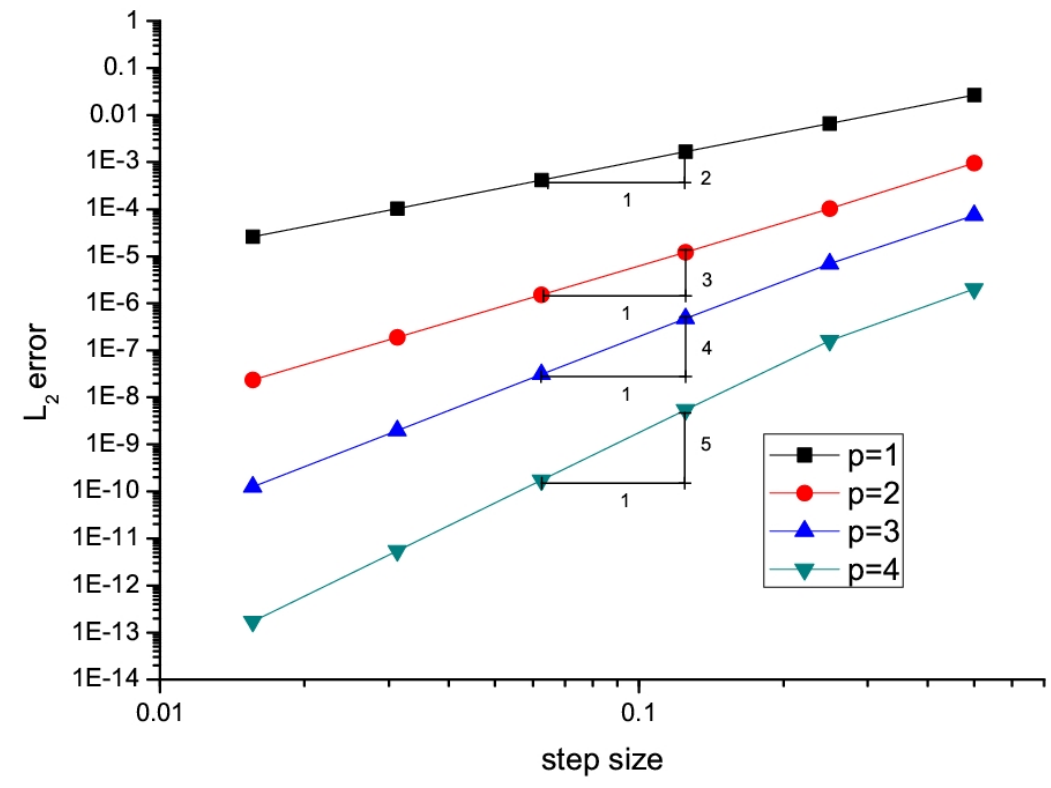

Figure 6: Convergence results for the catenary model. The $x$ coordinate represents the step size and the $y$ coordinate represents the $L_{2}$ error, which are shown in logarithmic form in base 10. Empirically, for each model described by splines with degree $p$, the order of convergence is $p+1$.

\section{2. "Tent"}

The tent model is inspired by vault roofs as shown in Reference [15]. Starting from an initial conceptual sketch of the shape, our algorithm turns it into a self-supporting surface. The boundary of the footprint is first drawn with CAD tools and then the design domain is modeled by NURBS patches. The layout of patches is shown in Fig. 4b. The self-supporting tent surface is designed by setting the stresses as $\sigma_{11}=1, \sigma_{22}=0.8$, and $\sigma_{12}=0$, and is shown in Fig. 7 .

\section{3. "Crest"}

The original "Crest" (Fig. 3) is an experimental sculpture designed by Zaha Hadid Architects. To construct it, the designers assembled very thin aluminium panels together into a flat plate and then lifted the plate into a self-supporting structure. The designers of "Crest" intend to investigate "the relationship between formal arrangement and structural performance"1, a topic with which we have affinity in this work. Inspired by the sculpture, using our algorithm, we create variations of self-supporting Crest models with different inner stress states. There is the pattern that the height of the surface increases when the ratio of stresses $\sigma_{22} / \sigma_{11}$ decreases. This pattern may help the architects to predict what shapes they will obtain, thus providing them a useful tool to explore possible designs. Fig. 8 shows three results for the Crest concept. The designers can choose the optimal design among them (and many others) according to their own aesthetics taste.

\footnotetext{
${ }^{1}$ According to the description in http://www.zaha-hadid.com/architecture/crest-installation/
} 
Table 1: Convergence tests of the catenary problem. The tolerance of the Newton iteration is $10^{-12}$.

\begin{tabular}{ccccc}
\hline Degree & Refine times & Cp per-side & $L_{2}$ error & Computation time(seconds) \\
\hline 1 & 1 & 5 & $2.678 \mathrm{E}-02$ & $2.150 \mathrm{E}-3$ \\
1 & 2 & 9 & $6.661 \mathrm{E}-03$ & $7.505 \mathrm{E}-3$ \\
1 & 3 & 17 & $1.659 \mathrm{E}-03$ & $3.687 \mathrm{E}-2$ \\
1 & 4 & 33 & $4.142 \mathrm{E}-04$ & $2.541 \mathrm{E}-1$ \\
1 & 5 & 65 & $1.034 \mathrm{E}-04$ & $2.753 \mathrm{E}+0$ \\
1 & 6 & 129 & $2.587 \mathrm{E}-05$ & $6.146 \mathrm{E}+1$ \\
\hline 2 & 1 & 7 & $9.479 \mathrm{E}-04$ & $5.070 \mathrm{E}-3$ \\
2 & 2 & 11 & $1.022 \mathrm{E}-04$ & $1.958 \mathrm{E}-2$ \\
2 & 3 & 19 & $1.220 \mathrm{E}-05$ & $9.809 \mathrm{E}-2$ \\
2 & 4 & 35 & $1.505 \mathrm{E}-06$ & $6.351 \mathrm{E}-1$ \\
2 & 5 & 67 & $1.875 \mathrm{E}-07$ & $5.461 \mathrm{E}+0$ \\
2 & 6 & 131 & $2.342 \mathrm{E}-08$ & $1.935 \mathrm{E}+2$ \\
\hline 3 & 1 & 9 & $7.430 \mathrm{E}-05$ & $1.126 \mathrm{E}-2$ \\
3 & 2 & 13 & $6.916 \mathrm{E}-06$ & $4.751 \mathrm{E}-2$ \\
3 & 3 & 21 & $4.784 \mathrm{E}-07$ & $2.527 \mathrm{E}-1$ \\
3 & 4 & 37 & $3.105 \mathrm{E}-08$ & $1.854 \mathrm{E}+0$ \\
3 & 5 & 69 & $1.975 \mathrm{E}-09$ & $3.106 \mathrm{E}+1$ \\
3 & 6 & 133 & $1.245 \mathrm{E}-10$ & $5.457 \mathrm{E}+2$ \\
\hline 4 & 1 & 11 & $2.040 \mathrm{E}-06$ & $2.578 \mathrm{E}-2$ \\
4 & 2 & 15 & $1.606 \mathrm{E}-07$ & $1.113 \mathrm{E}-1$ \\
4 & 3 & 23 & $5.405 \mathrm{E}-09$ & $5.243 \mathrm{E}-1$ \\
4 & 4 & 39 & $1.724 \mathrm{E}-10$ & $3.641 \mathrm{E}+0$ \\
4 & 5 & 71 & $5.436 \mathrm{E}-12$ & $1.038 \mathrm{E}+2$ \\
4 & 6 & 135 & $1.706 \mathrm{E}-13$ & $1.569 \mathrm{E}+3$ \\
\hline
\end{tabular}

\subsection{Top of Lilium tower: comparison with TNA}

The top of Lilium Tower is used for comparison with the TNA methods. Lilium tower is a building in Warsaw, Poland, also designed by Zaha Hadid Architects. In [9] a self-supporting mesh defined by 1201 vertices and 3504 edges approximating the top of Lilium tower is given. With our NURBS-based representation, only 36 control points are used, as shown in Fig. 9a. So compared with the TNA-based method using a discrete mesh surface, the shape computed by our method is smooth and the variables needed to describe the geometry are much fewer. As a result, our algorithm has better computational efficiency, as further discussed in section 5.1. In Masaaki et al.'s work [15], which also uses parametric surfaces for representing self-supporting surfaces, discrete self-supporting conditions, however, are still based on the equilibrium of the thrust network, which lacks the high order precision achieved with few variables that our isogeometric model provides.

\subsection{Trihole and Stadium}

The "Trihole" and "Stadium" models have complex topology. Our method handles them without difficulty. The input footprints are specified according to the top views of the mesh models 


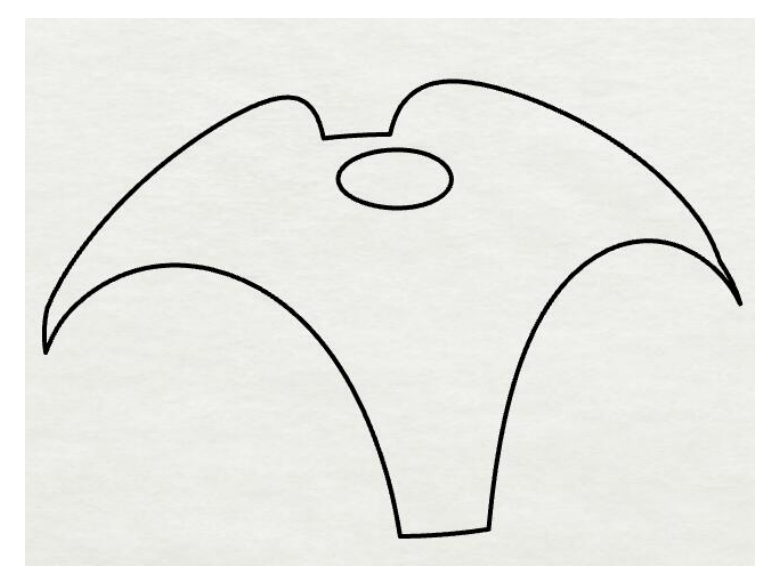

(a)

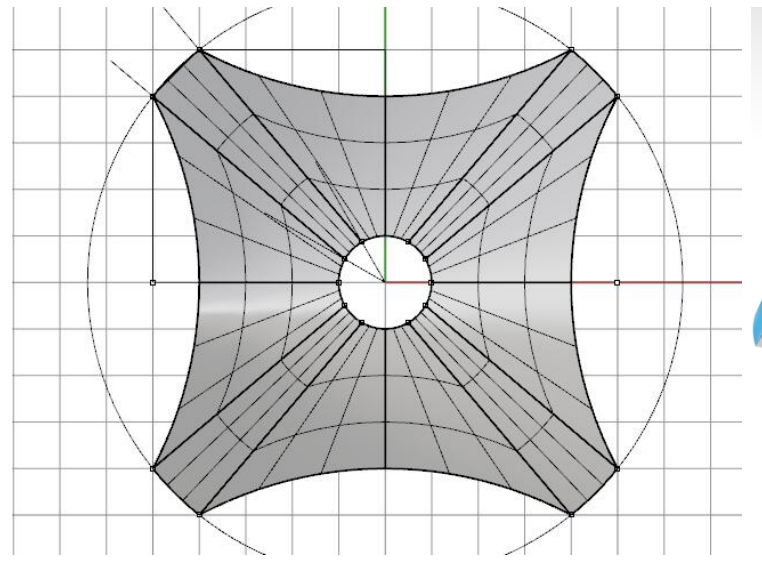

(c)

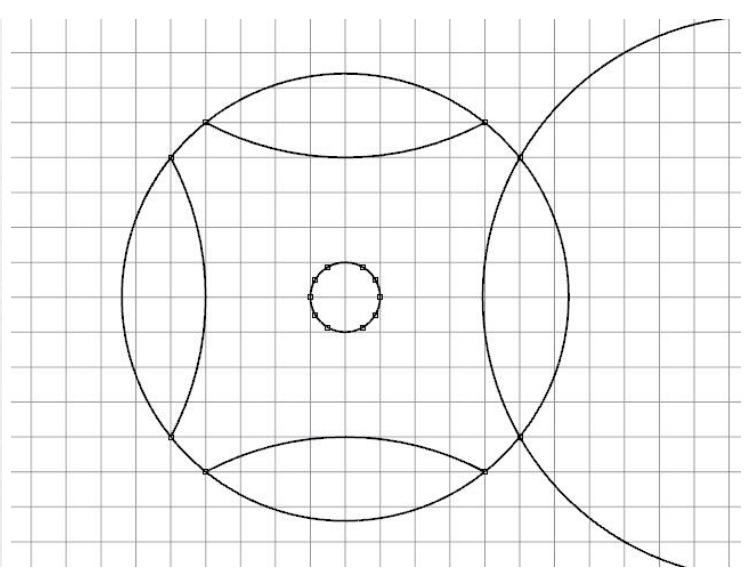

(b)

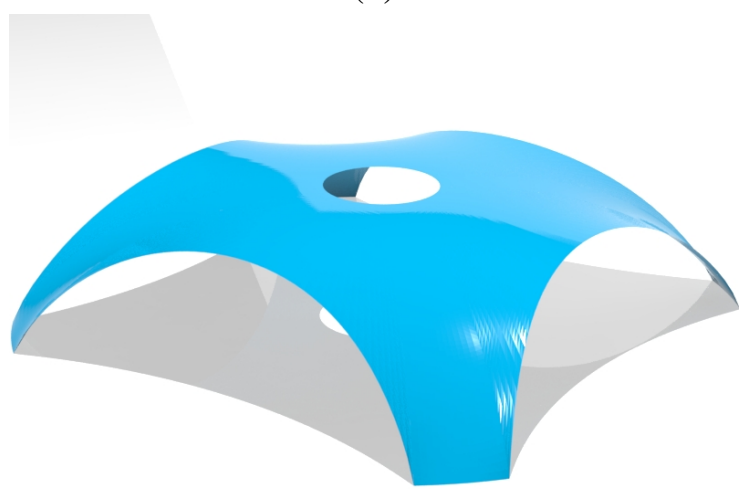

(d)

Figure 7: The design process of the tent model. (a) The design concept. (b) The input model is designed with CAD software. (c) The input model described by NURBS patches. (d) The output masonry structure.

shown in [8] and [9]; parts of the boundaries are fixed to the ground. Fig. 10 illustrates the computed self-supporting surfaces with our method.

\section{Discussions}

\subsection{Comparisons with TNA and IGA based methods}

We model masonry surfaces by parametric NURBS, which is suitable for describing smooth geometry. Compared with TNA-based methods, which use discrete graphs and meshes, the parametric description defines smooth surfaces with a smaller number of control points, thus enjoying a considerable advantage in computational efficiency. The runtime statistics of our algorithm are listed in Table 2. All the experiments are done on an Intel i7 processor clocked at $2.7 \mathrm{GHz}$. For example, the catenary and the crest models are defined with a single patch, thus the number of control points used to define the geometry is very small while the smooth shapes are well represented. Our algorithm finds the self-supporting shapes from the initial planar configurations within less than 0.1 seconds for both models. Complicated models with holes are represented using multiple 


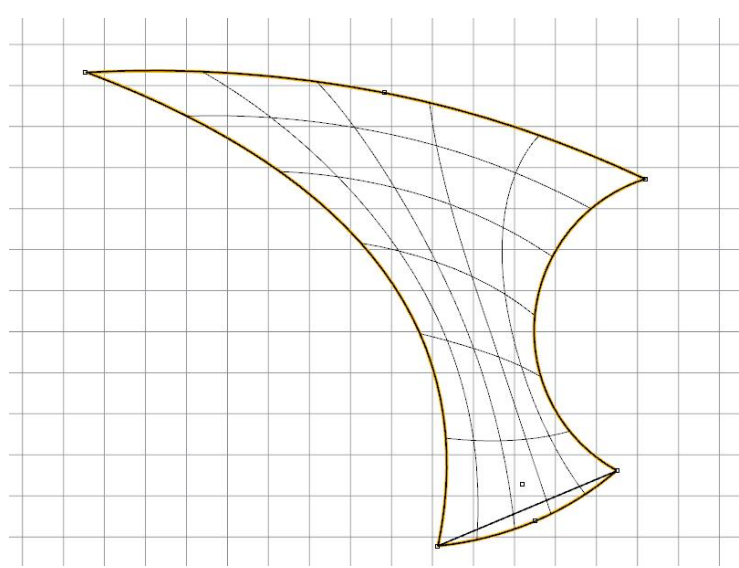

(a)

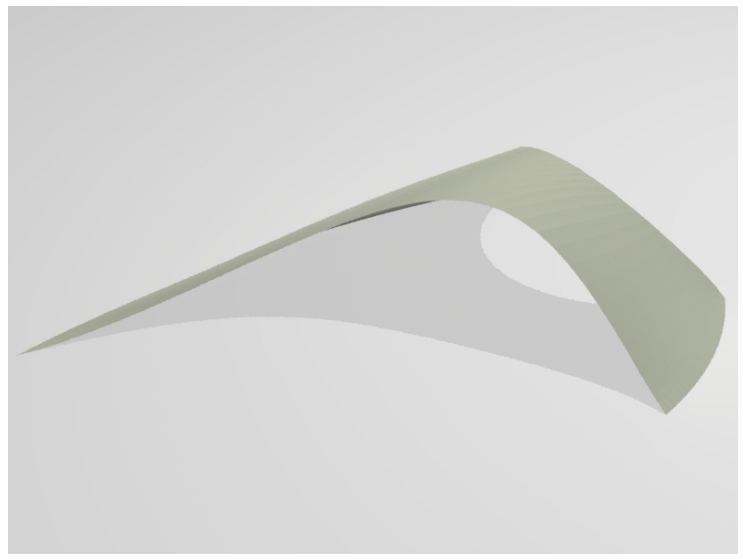

(c)

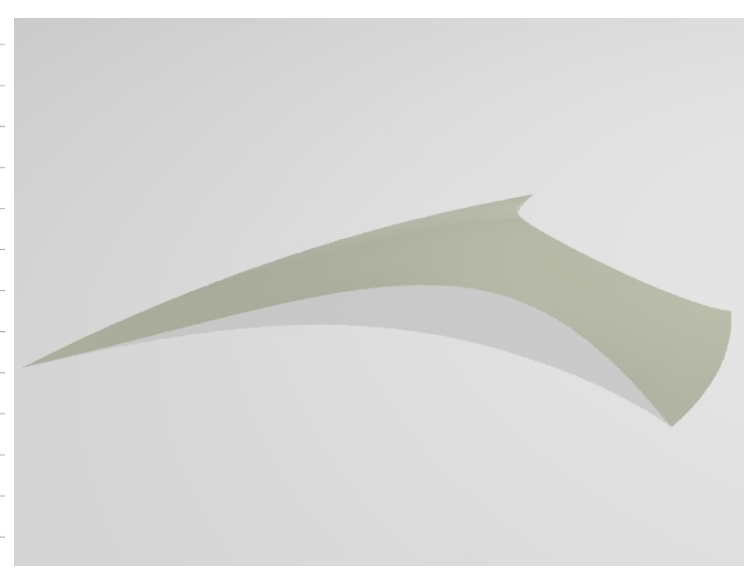

(b)

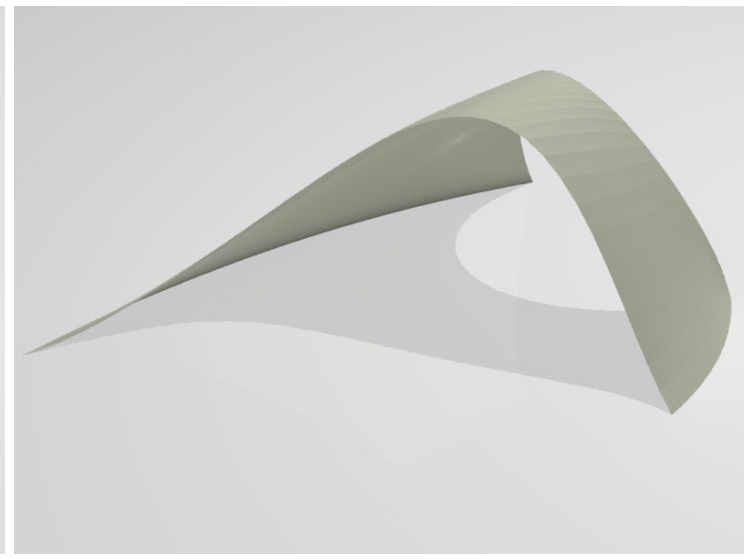

(d)

Figure 8: Crest model. (a) Free form curves and patch inspired by the "Crest" sculpture. The stresses are set as follows: $\sigma_{11}=-1, \sigma_{12}=0$ are used for all the examples. The other stresses are (b) $\sigma_{22}=-0.6$, (c) $\sigma_{22}=-0.3$, (d) $\sigma_{22}=-0.2$.

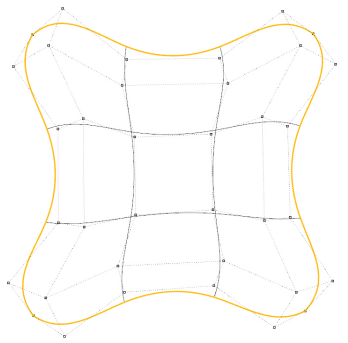

(a) Input geometry

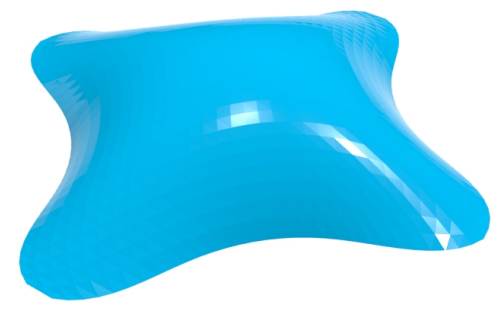

(b) Present result

Figure 9: Lilium tower. (a) The input geometry; (b) Result obtained by present algorithm with stresses as $\sigma_{11}=-0.5$, $\sigma_{22}=-0.5$, and $\sigma_{12}=0$. It is a continuous smooth surface described by NURBS, and the triangulation shown in the figure is only created by the rendering. 


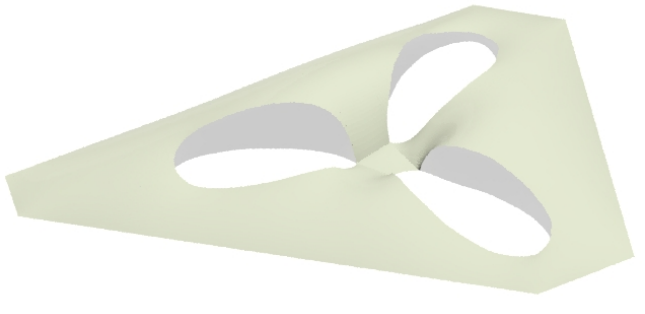

(a) Tri-hole model

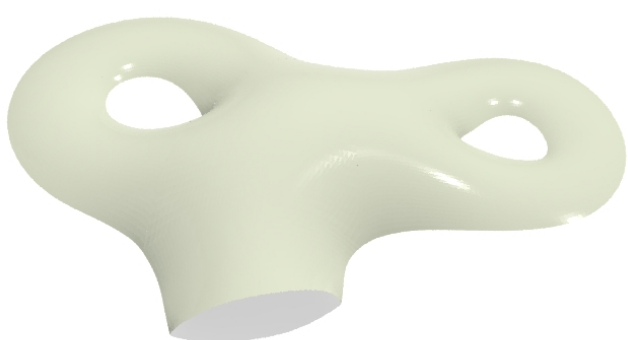

(b) Stadium

Figure 10: Two complex self-supporting surfaces computed by our method. (a) The tri-hole model has stresses as $\sigma_{11}=-0.2, \sigma_{22}=-1.0$, and $\sigma_{12}=0$. (b) The stadium model has stresses as $\sigma_{11}=-0.3, \sigma_{22}=-0.3$, and $\sigma_{12}=0$.

NURBS surface patches, as in the examples of the tent, the top of lilium tower, and the tri-hole models. Adjacent patches share the control points along their common boundary, so that the surface has $C^{0}$ continuity. Again our algorithm can effectively find the self-supporting shapes, with computation time only about $25 \%$ of that by TNA in [9].

Table 2: Computational statistics of our algorithm.

\begin{tabular}{ccccc}
\hline Fig. & Con. Pts. & Iters. & Err. & Time (s) \\
\hline $5 \mathrm{c}$ & 25 & 12 & $10^{-12}$ & 0.027 \\
7 & 828 & 4 & $10^{-12}$ & 2.069 \\
8 & 56 & 4 & $10^{-12}$ & 0.093 \\
$9 \mathrm{~b}$ & 36 & 4 & $10^{-12}$ & 0.044 \\
$10 \mathrm{~b}$ & 1158 & 4 & $10^{-12}$ & 4.218 \\
\hline
\end{tabular}

Table 3: Comparison of computational efficiency. The termination condition is that the tolerance of the Newton iteration is $10^{-12}$. For a simple shape that can be described by a single patch, our method can obtain a self-supporting shape in real time (less than 0.1 seconds). For whole shapes which must be described by multi-patch geometry, the present algorithm only needs about $25 \%$ time of that when using TNA.

\begin{tabular}{ccccccc}
\hline \multirow{2}{*}{ Fig. } & \multicolumn{3}{c}{ Our algorithm } & \multicolumn{3}{c}{ Ref. [9] } \\
\cline { 2 - 7 } & Con. Pts. & Iters & Time (s) & Vertices & iterations & Time (s) \\
\hline $9 \mathrm{~b}$ & 36 & 4 & 0.044 & 1201 & 9 & 21.6 \\
$10 \mathrm{~b}$ & 1158 & 4 & 4.218 & 1535 & 21 & 17.0 \\
\hline
\end{tabular}

Alic and Persson's dynamic relaxation method [30] and Philipp et al.'s updated reference strategy [28] also use isogeometric analysis for the computation of membrane structures. In these two papers the form-finding approach is used to compute architectural surfaces. Our method is more practical and effective for the design of masonry shapes. In particular, one advantage is that our algorithm does not need a specific input shape as the target, and useful self-supporting shapes 
can be obtained efficiently by tuning the inner stress states. The form finding methods follow the reverse design approach: an existing model must be used as the input and the outputs are all in similar shapes with the input. Another advantage is the computational efficiency: our method applies Newton's method to handle the nonlinear loads and converges within very few (typically about 5) iterations. The results in Table 3 show that form finding methods generally take more computation time.

\subsection{Influence of Airy stress}

In this section, we explore the influence of Airy stress in tuning the self-supporting shape. To carry out the analysis, we use a simple square domain of $[-1,1] \times[-1,1]$ on which self-supporting surfaces are defined.

\subsubsection{Scale and ratio}

In actual masonry buildings, gravity loads vary from dozens to thousands of pascals depending on the building material, while geometric scales range from meters to dozens of meters. According to the control formula in Eq. 10, the relative ratio of Airy stress to the gravity load determines the shape. Therefore, without loss of generality we assume a unit gravity load, and fix all four boundaries of the surface to the ground. We explore the effect of uniform Airy stress functions which have zero shear stress $\left(\sigma_{12}=\sigma_{21}=0\right)$ and varying normal stresses $\sigma_{11}, \sigma_{22}$. In addition, we always set $\sigma_{11}>\sigma_{22}$, because models with $\sigma_{11}<\sigma_{22}$ can be obtained simply by rotation. Our results show that the feasible design domain of Airy stresses under this setting locates within a triangle, as plotted in Fig. 11. The main conclusions from the test are as follows. Through this example, it is shown that the meaningful values for the Airy stresses reside in a well-defined region and the obtained shapes are predictable by tuning the values.

a) When both stresses are tiny, which means the structure is in a loose state and there is not enough compression force to hold the pieces together, Newton's iteration will not converge and no self-supporting shapes can be obtained.

b) When stress increases, the height of the obtained shape generally decreases. This phenomenon is explained by the fact that equilibrium with smaller plane stresses can be obtained with steeper shapes, until reaching the extreme case when walls are built straight up with blocks. In the opposite direction, when inner stress increases, the height of the obtained shape decreases, until it becomes planar in the extreme situation.

c) When the ratio of two normal stresses $\sigma_{11}, \sigma_{22}$ increases, the shape cross sections in the two directions will be more different, as shown in Fig. 12.

d) The feasible design domain in this problem is located within a triangle with vertexes $(0.3,0.3)$, $(1.49,1.49)$ and $(3.2,0.002)$. A symmetrical triangle plotted by the dashed line is the feasible domain for $\sigma_{11}<\sigma_{22}$. 


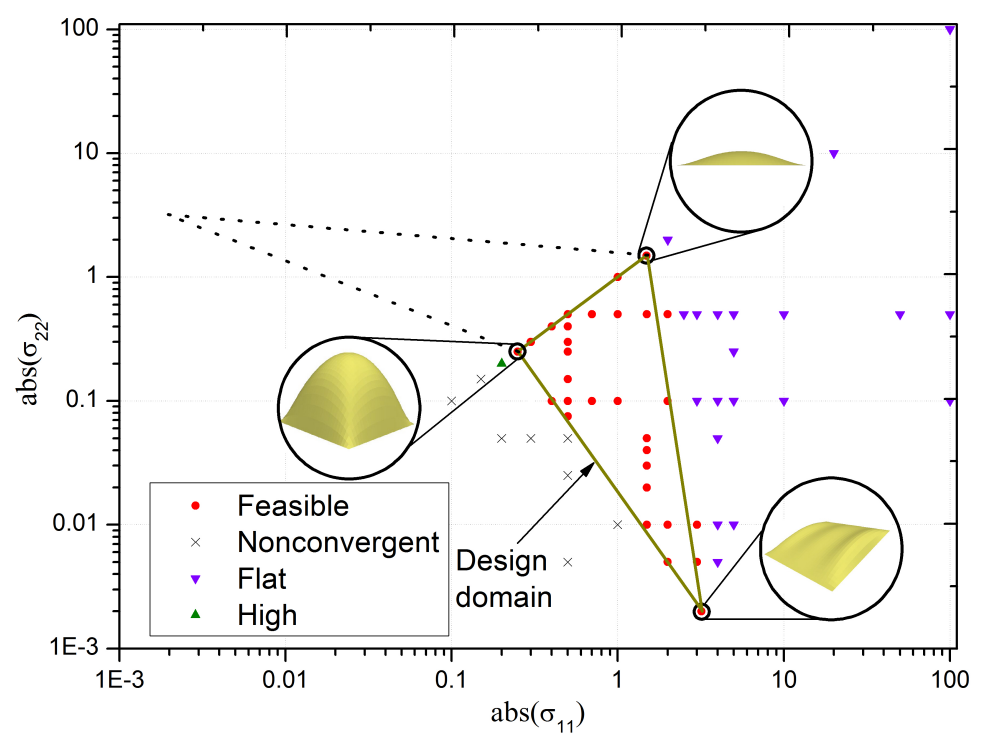

Figure 11: The feasible design domain of Airy stresses. Shear stresses are set to zero, and the absolute value of normal stresses are plotted in logarithmic-logarithmic coordinates. The red circular spots represent feasible values for obtaining self-supporting shapes. The cross spots mean that the Newton iteration does not converge. The inverted triangles represent flat shapes that are not interesting, while the triangles represent cliffy shapes. The values are chosen by practical experiences that shapes with a maximum height less than 0.2 are deemed flat, and more than 4 as high.
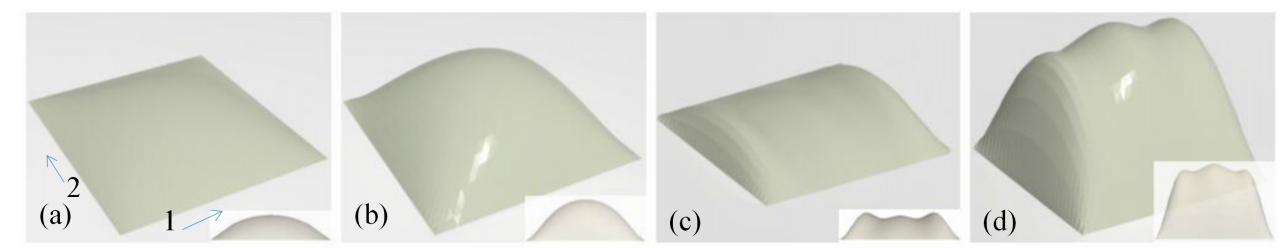

Figure 12: The influence of the scale of Airy stress functions on self-supporting shapes. The insets on down-right corners show the side views of the shapes. For all models $\sigma_{12}=\sigma_{21}=0$. (a) $\sigma_{11}=\sigma_{22}=-1$, (b) $\sigma_{11}=\sigma_{22}=-0.5$, (c) $\sigma_{11}=-2, \sigma_{22}=-0.01$, (d) $\sigma_{11}=-0.5, \sigma_{22}=-0.1$.

\subsubsection{Propagation of boundary conditions}

In this section, a square domain with two opposite and curved boundaries is used to study the influence of boundary conditions. A model with four patches is used. The boundary curve is given by the function $0.3|\sin (\pi x)|$, as shown in Fig. 13. The $L_{2}$ projection method is used to force the boundary of the model to keep the same shape as the boundary curve.

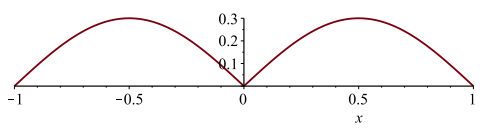

Figure 13: The boundary curve.

First, a sequence of different stresses with zero shear force and corresponding results are shown in Fig. 14. We see that when the normal stress $\sigma_{22}$ is small, the influence of the curved boundary 
is limited to the sides and the interior domain is not affected; with the increase of $\sigma_{22}$, however, the influence propagates and can even dominate the whole shape.

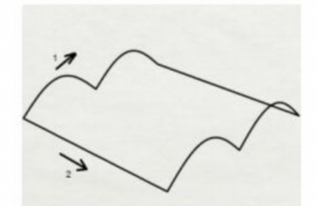

(a)

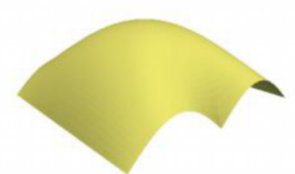

(b)

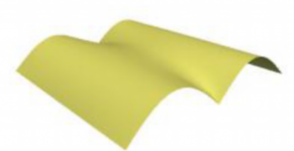

(c)

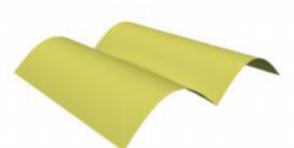

(d)

Figure 14: Influence of the boundary increases with the corresponding Airy stress values. Curved boundary conditions shown in Fig. 13 are applied on two opposite sides of the square. The other two sides are pinched, with a constant normal stress $\sigma_{11}=-0.5$. The absolute value of normal stresses on the wiggly sides $\sigma_{22}$ gradually increase. (a) the input boundaries, (b) $\sigma_{22}=-0.5$, (c) $\sigma_{22}=-5$, (d) $\sigma_{22}=-50$. Shape construction in (d) is difficult, because the inner stress is large and huge force must be applied on the boundary to maintain the stress state, which is not practical in construction.

To investigate the propagation direction of the boundary conditions under the influence of stress functions, we further conduct the following tests. According to theory of elasticity, the two main stresses within a membrane can be computed as follows:

$$
\left.\begin{array}{l}
\sigma^{1} \\
\sigma^{2}
\end{array}\right\}=\frac{\sigma_{11}+\sigma_{22}}{2} \pm \sqrt{\left(\frac{\sigma_{11}-\sigma_{22}}{2}\right)^{2}+\sigma_{12}^{2}}
$$

The two main stresses are both negative; the one with the larger absolute value is denoted as $\sigma^{1}$. The angle between the main stress $\sigma^{1}$ and the $x$ coordinate axis is denoted as $\alpha^{1}$, and

$$
\tan \left(\alpha^{1}\right)=\frac{\sigma^{1}-\sigma_{11}}{\sigma_{12}} .
$$

Five stress states are picked from Mohr's stress circle with main stresses $\sigma^{1}=-50, \sigma^{2}=-0.5$. The angles $\alpha^{1}$ of the five states are $90^{\circ}, 60^{\circ}, 45^{\circ}, 30^{\circ}$ and $0^{\circ}$. In Fig. 15, the top view of the obtained shapes are plotted so that the propagation of curved boundary condition can be clearly observed. The results show that the propagation direction of boundary conditions coincides with the direction of main stress. This result is very useful for improving our understanding about the behavior of masonry surfaces.

\subsection{Influence of parameterizations}

The influence of parameterization for multi-patch models is tested. Fig. 16 shows two parametric models for an input shape with the same planar boundary: the left model has four patches, while the right model has three patches. The outer boundaries of the models are fixed to the ground and the central circular boundary rises to form self-supporting surfaces. The Airy stress is set as $\sigma_{11}=\sigma_{22}=-0.4, \sigma_{12}=0$. The results show that differences in the parametric models do not significantly affect the final shapes obtained using our algorithm. The computed masonry shapes from these two parameterizations are almost the same, with their Hausdorff distance being $0.68 \%$ of the bounding box diagonal length of the result shapes. 

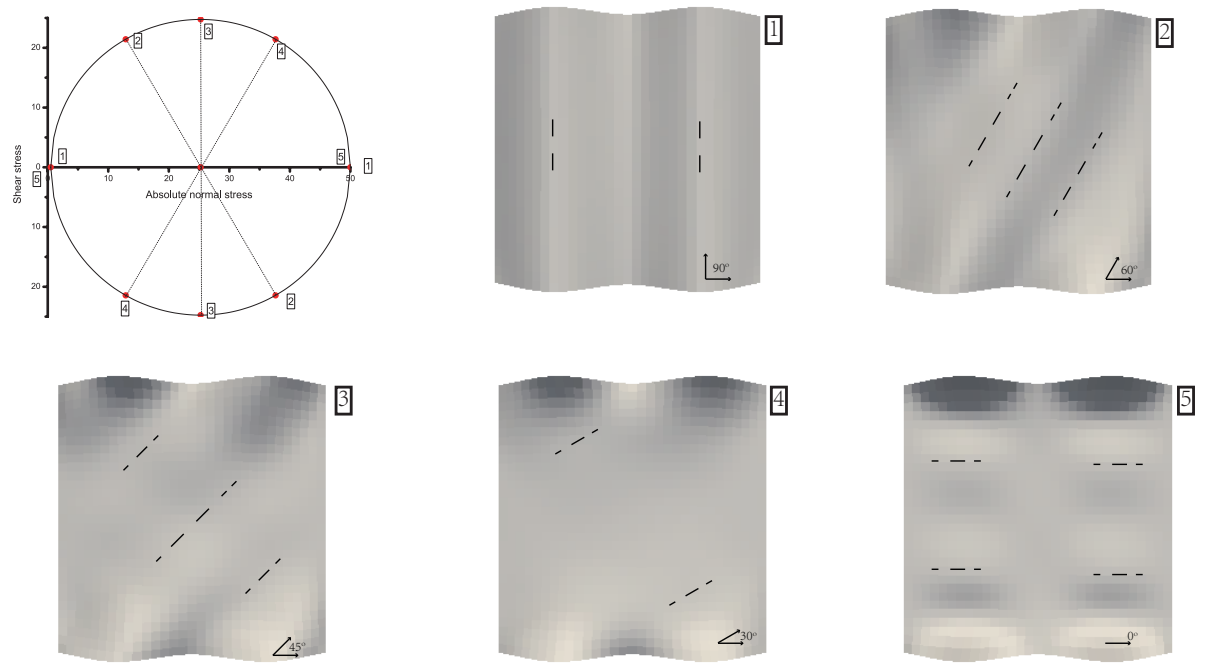

Figure 15: Self-supporting shapes corresponding to the five stress states picked from the Mohr's stress circle. The main stress direction coincides with the propagation direction of applied boundary conditions.

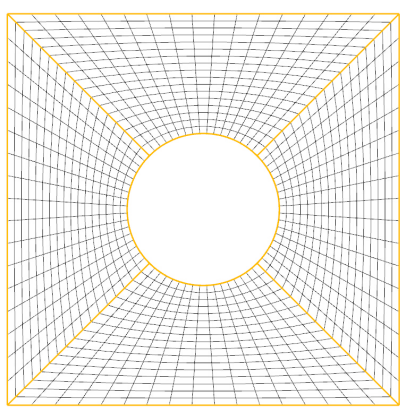

(a) Input model 1

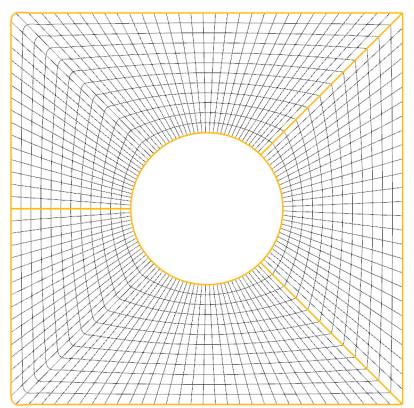

(b) Input model 2

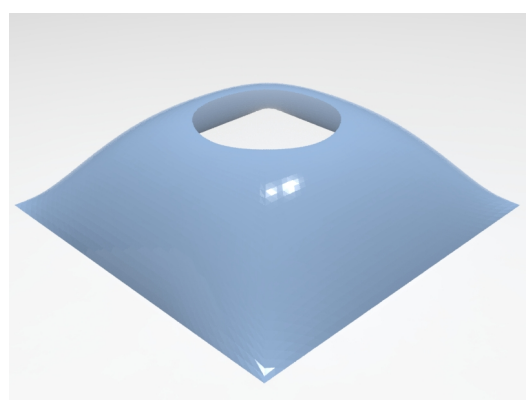

(c)

Figure 16: Two different parametric methods for the "plate-hole" model with the same geometrical shape. Our algorithm obtains stable results for these two parametric models. (a) Input model with four patches; (b) Input model with three patches; (c) The obtained shapes are close to one another and cannot be visually distinguished.

\subsection{Exponential Airy stress}

Constant stress states have been used in previous examples so far. However, Airy stress functions are not restricted to constant stress states. In this section we show an example with varying stress states. In particular, exponential functions make a qualified candidate for Airy stress function because of their strict convexity. For example, the function

$$
\Phi=-\left(e^{x}+e^{y}\right)
$$

is concave and thus the self-supporting condition is always satisfied. In this case, the stress tensor takes the form

$$
\sigma=\left[\begin{array}{cc}
-e^{x} & 0 \\
0 & -e^{y}
\end{array}\right] .
$$


The shape of the Airy stress function is shown in Fig. 17(a). The self-supporting shape is shown together with the corresponding Airy stress in Fig. 17(b), and in different views in Fig. 17(c) and 17(d). This example demonstrates the ability of our algorithm to deal with various types of Airy stress functions.

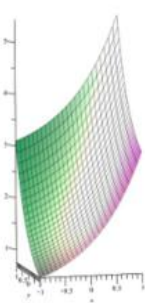

(a)

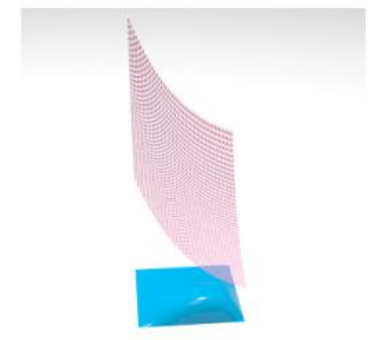

(b)

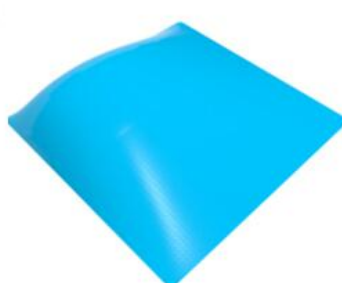

(c)

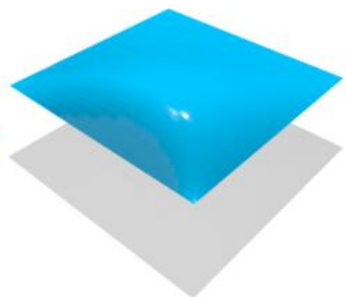

(d)

Figure 17: Computational results obtained for an exponential Airy stress. (a) Absolute value of the Airy function. (b) Obtained shape and corresponding Airy function. (c) and (d) show the shape in different perspectives.

\section{Conclusion}

In this paper, self-supporting masonry surfaces are modeled with NURBS based IGA and a heuristic strategy for designing the surfaces is proposed. The equilibrium equation for selfsupporting surfaces is derived from membrane shell theory and can be effectively solved by the IGA representation. Nonlinear equations that consider changing gravity loads with shapes are solved via Newton's method. The convergence rate of the presented algorithm is shown to be one order higher than the degree of applied NURBS basis functions. Airy stress states within the shape can be manipulated and the corresponding self-supporting surfaces are efficiently calculated. Moreover, the Airy stress space for meaningful designs is explored and discussed, which provides guidance for design. Various self-supporting structures with complex topology and shapes are demonstrated. These shapes can help architects to find the ideal conceptual design, and to get an idea of the stress states within the design. Overall, the proposed algorithm accurately and efficiently calculates the shapes of self-supporting surfaces and provides an effective tool for design.

There are some limitations of the present method. The stress state of masonry structure is wider than that described by Airy stress function, because divergence free stress tensors exist that are not second-order differentials of a function. In future works, the algorithm to solve the general differential equation of self-supporting masonry using isogeometric analysis, without the aid of Airy stress function can be investigated. The present algorithm focuses on the elastic deformation and the plastic deformation can be embedded into the present strategy in the future work for the evaluation of existing masonry structures. Higher continuity such as $C^{1}$ smoothness along the boundary of the adjacent patches of complex structure can also be considered. 


\section{Acknowledgement}

We would like to thank Prof. Jiaye Wang for the valuable comments and Dr. Etienne Vouga for kindly sharing the code of the thrust network method. This work is funded by National Natural Science Foundation of China (No. 11702056, 61572021), Fundamental Research Funds for the Central Universities (No. DUT17JC32).

\section{Reference}

[1] D. L. Lopez, T. Van Mele, P. Block, Tile vaulting in the 21st century, Informes de la Construccion 68 (544) (2016) 1-9.

[2] G. Poleni, Memorie istoriche della gran cupola del tempio vaticano: e de' danni di essa, e de' ristoramenti loro, divise in libri cinqve, Stamperia del Seminario, 1748.

[3] P. Block, M. DeJong, J. Ochsendorf, As hangs the flexible line: Equilibrium of masonry arches, Nexus Network Journal 8 (2) (2006) 13-24.

[4] P. Roca, M. Cervera, G. Gariup, L. Pela, Structural analysis of masonry historical constructions. classical and advanced approaches, Archives of Computational Methods in Engineering 17 (3) (2010) 299-325.

[5] S. Huerta, Mechanics of masonry vaults: the equilibrium approach, Historical Constructions (2001) 47-70.

[6] P. Block, T. Ciblac, J. Ochsendorf, Real-time limit analysis of vaulted masonry buildings, Computers and Structures 84 (29) (2006) 1841-1852. doi:10.1016/j.compstruc.2006.08.002.

[7] P. Block, Thrust network analysis: Exploring three-dimensional equilibrium, Ph.D. thesis, Massachusetts Institute of Technology (2009).

[8] D. Panozzo, P. Block, O. Sorkine-Hornung, Designing unreinforced masonry models, ACM Transactions on Graphics (TOG) 32 (4) (2013) 91.

[9] E. Vouga, M. Hobinger, J. Wallner, H. Pottmann, Design of self-supporting surfaces, ACM Transactions on Graphics (TOG) 31 (4) (2012) 87.

[10] Y. Liu, H. Pan, J. Snyder, W. Wang, B. Guo, Computing self-supporting surfaces by regular triangulation, ACM Transactions on Graphics (TOG) 32 (4) (2013) 1-10.

[11] M. Deuss, D. Panozzo, E. Whiting, Y. Liu, P. Block, O. Sorkine-Hornung, M. Pauly, Assembling self-supporting structures, ACM Transactions on Graphics (TOG) 33 (6) (2014) 214:1-214:10.

[12] K.-U. Bletzinger, R. Wüchner, F. Daoud, N. Camprubí, Computational methods for form finding and optimization of shells and membranes, Computer Methods in Applied Mechanics and Engineering 194 (30) (2005) 3438-3452. doi:10.1016/j.cma.2004.12.026.

[13] J. Jordan, K.-U. Bletzinger, Efficient simulation of large masonry structures with discrete crack models, Bauingenieur 86 (2011) 520-528.

[14] K.-U. Bletzinger, E. Ramm, Computational form finding and optimization, in: S. Adriaenssens, P. Block, D. Veenendaal, C. Williams (Eds.), Shell Structures for Architecture: Form Finding and Optimization, Taylor \& Francis Group, 2014, Ch. 5, pp. 43-55.

[15] M. Miki, T. Igarashi, P. Block, Parametric self-supporting surfaces via direct computation of airy stress functions, ACM Transactions on Graphics (TOG) 34 (4) (2015) 89:1-89:12.

[16] M. Dhanasekar, A. Page, P. Kleeman, The failure of brick masonry under biaxial stresses, in: ICE Proceedings, Vol. 79, Thomas Telford, 1985, pp. 295-313.

[17] L. Zhang, Q. Gao, H. W. Zhang, An efficient algorithm for mechanical analysis of bimodular truss and tensegrity structures, International Journal of Mechanical Sciences 70 (1) (2013) 57-68. doi:10.1016/j.ijmecsci.2013.02.002.

[18] Z. Du, X. Guo, Variational principles and the related bounding theorems for bi-modulus materials, Journal of the Mechanics and Physics of Solids 73 (1) (2014) 183-211. doi:10.1016/j.jmps.2014.08.006.

[19] M. Giaquinta, E. Giusti, Researches on the equilibrium of masonry structures, Archive for Rational Mechanics and Analysis 88 (4) (1985) 359-392.

[20] D. O’Dwyer, Funicular analysis of masonry vaults, Computers and Structures 73 (1) (1999) 187-197. 
[21] T. J. R. Hughes, J. A. Cottrell, Y. Bazilevs, Isogeometric analysis: CAD, finite elements, NURBS, exact geometry and mesh refinement, Computer Methods in Applied Mechanics and Engineering 194 (39-41) (2005) 4135-4195.

[22] D. J. Benson, Y. Bazilevs, M. C. Hsu, T. J. R. Hughes, Isogeometric shell analysis: The Reissner-Mindlin shell, Computer Methods in Applied Mechanics and Engineering 199 (5-8) (2010) 276-289.

[23] M. Breitenberger, A. Apostolatos, B. Philipp, R. Wüchner, K. U. Bletzinger, Analysis in computer aided design: Nonlinear isogeometric B-rep analysis of shell structures, Computer Methods in Applied Mechanics and Engineering 284 (2015) 401-457. doi:10.1016/j.cma.2014.09.033.

[24] K. Sommerwerk, M. Woidt, M. C. Haupt, P. Horst, Reissner-Mindlin shell implementation and energy conserving isogeometric multi-patch coupling, International Journal for Numerical Methods In Engineering 109 (7) (2017) 982-1012.

[25] A. Cazzani, M. Malagu, E. Turco, Isogeometric analysis: a powerful numerical tool for the elastic analysis of historical masonry arches, Continuum Mechanics and Thermodynamics 28 (1-2) (2016) 139-156. doi:10.1007/s00161-014-0409-y.

[26] B. Philipp, M. Breitenberger, R. Wüchner, K.-U. Bletzinger, Design of architectural membranes with isogeometric elements, 11th World Congress on Computational Mechanics (2014) 303-314.

[27] B. Philipp, R. Wüchner, K.-U. Bletzinger, Advances in the form-finding of structural membranes, Procedia Engineering 155 (2016) 332-341.

[28] B. Philipp, M. Breitenberger, I. Dauria, R. Wüchner, K. U. Bletzinger, Integrated design and analysis of structural membranes using the Isogeometric B-Rep Analysis, Computer Methods in Applied Mechanics and Engineering 303 (2016) 312-340. doi:10.1016/j.cma.2016.02.003.

[29] A. M. Bauer, M. Breitenberger, B. Philipp, R. Wüchner, K. U. Bletzinger, Embedded structural entities in nurbsbased isogeometric analysis, Computer Methods in Applied Mechanics and Engineering 325 (2017) 198-218. doi:10.1016/j.cma.2017.07.010.

[30] V. Alic, K. Persson, Form finding with dynamic relaxation and isogeometric membrane elements, Computer Methods in Applied Mechanics and Engineering 300 (2016) 734-747. doi:10.1016/j.cma.2015.12.009.

[31] V. S. Kelkar, R. T. Sewell, Fundamentals of the Analysis and Design of Shell Structures, Prentice Hall, 1987.

[32] M. Angelillo, E. Babilio, A. Fortunato, Singular stress fields for masonry-like vaults, Continuum Mechanics and Thermodynamics 25 (2-4) (2013) 423-441. doi:10.1007/s00161-012-0270-9.

[33] J. Jerome J. Connor, S. Lamar, J. P. Wolf, Automatic solution of Pucher's Equation, Report, Massachusetts Institute of Technology (1965).

[34] S. Timoshenko, J. N. Goodier, Theory of Elasticity, McGraw-Hill Book Company, New York, 1951.

[35] R. Abraham, J. E. Marsden, T. Ratiu, Manifolds, tensor analysis, and applications, Vol. 75, Springer Science \& Business Media, 2012.

[36] M. Kapl, V. Vitrih, B. Jüttler, K. Birner, Isogeometric analysis with geometrically continuous functions on two-patch geometries, Computers \& Mathematics with Applications 70 (7) (2015) 1518-1538. doi:10.1016/j.camwa.2015.04.004.

[37] M. Kapl, F. Buchegger, M. Bercovier, B. Jüttler, Isogeometric analysis with geometrically continuous functions on planar multi-patch geometries, Computer Methods in Applied Mechanics and Engineering 316 (2017) 209234. doi:10.1016/j.cma.2016.06.002.

[38] K. Birner, B. Jüttler, A. Mantzaflaris, Bases and dimensions of C1-smooth isogeometric splines on volumetric two-patch domains, Graphical Models 99 (2018) 46-56. doi:10.1016/j.gmod.2018.08.001. 